\title{
STRONG REGULARITY IN ARBITRARY RINGS
}

\author{
TETSUO KANDÔ
}

An element $a$ of a ring $R$ is called regular, if there exists an element $x$ of $R$ such that $a x a=a$, and a two-sided ideal $a$ in $R$ is said to be regular if each of its elements is regular. B. Brown and N. H. McCoy [1] has recently proved that every ring $R$ has a unique maximal regular two-sided ideal $M(R)$, and that $M(R)$ has the following radical-like property: (i) $M(R / M(R))=0$; (ii) if $a$ is a two-sided ideal of $R$, then $M(a)=a \cap M(R)$; (iii) $M\left(R_{n}\right)=(M(R))_{n}$, where $R_{n}$ denotes a full matrix ring of order $n$ over $R$. Arens and Kaplansky [2] has defined an element $a$ of $R$ to be strongly regular when there exists an element $x$ of $R$ such that $a^{2} x=a$. We shall prove in this note that replacing "regularity" by "strong regularity," we have also a unique maximal strongly regular ideal $N(R)$, and shall investigate some of its properties.

1. Existence and properties of $N(R)$.

The existence proof of $N(R)$ can be accomplished along the line of Brown and McCoy [1].

Definition 1. An element' $a$ of a ring $R$ is called strongly regular, if and only if there exists an element $x$ of $R$ such that $a^{2} x=a$. A (two-sided) ideal $a$ in $R$ is called a strongly regular ideal, if each of its element is strongly regular. Finally, we call an element $a \in R$ properly strongly regular, if the principal ideal $(a)$ generated by $a$ is strongly regular.

LEMMA 1. If $a^{2} y-a$ is strongly regular, so is a too.

Proof. By virtue of strong regularity of $a^{2} y-a$, there exists an element $z$ such that $\left(a^{2} y-a\right)^{2} z=a^{2} y-a$. Setting $x=y-z+a y z+y a z-y a^{2} y z$, we have readily $a^{2} x=a$.

LEMMA 2. The set $N(R)$ of all properly strongly regular elements of $R$ is a strongly regular ideal.

Proof. That $z \in N(R)$ and $t \in R$ implies $(z t) \subset N(R)$ whence $z t \in N(R)$; similarly, $t z \in N(R)$. On the other hand, let $z_{1}$ and $z_{2}$ be any elements of $N(R)$ and let $a \in\left(z_{1}-z_{2}\right)$. Then we have $a=u_{1}-u_{2}$, where $u_{i} \in\left(z_{i}\right)$. By strong regularity of $\left(z_{1}\right)$, we have $u_{1}^{2} r=u_{1}$ for some $r \in R$. Then $a^{2} r-a=\left(u_{1}-u_{2}\right)^{2} r$ $-\left(u_{1}-u_{2}\right)=u_{2}+u_{2}^{2} r-u_{1} u_{2} r-u_{2} u_{1} r \in\left(u_{2}\right) \subset\left(z_{2}\right)$, and $a^{2} r-a$ is strongly regular. Then, Lemma 1 implies that $a$ is strongly regular, and the proof is complete.

Received September 1, 1951. 
From Lemma 2, we have immediately

THEOREM 1. Every ring $R$ has a unique maximal strongly regular ideal $N(R)$.

THEOREM 2. For every ring $R$ we have $N(R / N(R))=0$.

Proof. Let us denote by $\bar{a}$ the residue class $\bmod N(R)$ containing $a$. Suppose that $\bar{b} \in N(R / N(R))$ and $a$ is any element of $(b)$. Then $\bar{a}$ is as an element of $(\bar{b})$ strongly regular in the ring $R / N(R): \bar{a}^{2} \bar{x}=\bar{a}$, that is $a^{2} x-a \in N(R)$, and hence $a^{2} x-a$ is strongly regular. It follows therefore from Lemma 1 that $a$ is strongly regular. Since we have proved that every element of the ideal $(b)$ is strongly regular, we have $b \in N(R)$, i.e. $\bar{b}=0$.

Lemma 3. Let a be a two-sided ideal of $R$. Then, an element a of a is properly strongly regular in the ring $a$ if and only if it is strongly regular in the ring $R$.

Proof. Let $a$ be properly strongly regular in $a$, and let $b$ be any element of the ideal ( $a$ ) generated by $a$ in $R$. Then, we have $b=n a+u a+a v+\sum u_{i} a v_{i}$, where $n$ is an integer and $u$ 's and $v$ 's are elements of $R$. Since $a$ is strongly regular, there exists an element $x \in \mathfrak{a}$ such that $a^{2} x=a$. Consequently, $b=n a$ $+(u a) a x+a(a x v)+\sum\left(u_{i} a\right) a\left(x v_{i}\right), u a, a x v, u_{i} a, x v_{i} \in a$. Hence we have $b \in(a)^{\prime}$, where $(a)^{\prime}$ denotes an ideal generated by $a$ in $a$. Therefore, $b$ is strongly regular, and the element $a$ is properly strongly regular in $R$. The converse part is clear.

From Lemma 3, we have immediately

Theorem 3. If a is a two-sided ideal in $R$, then $N(a)=a \cap N(R)$.

2. Some relations between $N(R)$ and $M(R)$.

Let us consider some properties of elements in $N(R)$.

LEMMA 4. $N(R)$ has no non-zero nilpotent element.

Proof. Let $a \in N(R)$, and $a^{n}=0$. Then $a^{2} x=a$, and so $a=a^{2} x=\ldots$ $=a^{n} x^{n-1}=0$.

Lemma 5. Let $a \in N(R)$ and $x$ be an element in $R$ such that $a^{2} x=a$. Then, (i) $a^{2} x=a x a=x a^{2}=a$, and $a$ is regular. (ii) $a x=x a$, and $a x$ is an idempotent. (iii) $e=a x$ belongs to the center of $R$.

Proof. From $a^{2} x=a$, we have easily $(a-a x a)^{2}=0$. Since $a-a x a \in N(R)$. Lemma 4 implies $a=a x a$, and similarly $a x a=x a^{2}$, so we have (i). From $a x$ $=\left(x a^{2}\right) x=x\left(a^{2} x\right)=x a$ we have (ii). As for (iii), let $u$ be any element of $R$. By analogous argument as (i), we have $u e=e u e, u e=e u e$, and therefore $u e=e u$.

The above lemma shows that each element of $N(R)$ is regular, so we have

THEOREM 4. $N(R) \subset M(R)$. 
While $M(R)$ satisfies $M\left(R_{n}\right)=(M(R))_{n}$ (cf. [1]), $N(R)$ does not possess this property, which is shown by the following theorem:

Theorem 5. Let $R_{n}$ be the full matrix ring of order $n>1$ over $R$. Then, $N\left(R_{n}\right)=0$.

Proof. First, let us suppose that $R$ has a unit element. Let $A \in N\left(R_{n}\right)$. Then there exists a matrix $X$ such that $A^{2} X=A$, and $A X$ belongs to the center of $R_{n}$. Hence, $A X=a E$, where $a$ is an element belonging to the center of $R$, and $E$ is the unit matrix of $R_{n}$. So, we have $A=a A$, and

$$
B=\left(\begin{array}{ccccc}
0 & a & 0 & \ldots & 0 \\
0 & 0 & 0 & \ldots & 0 \\
\ldots & \ldots & \ldots & \ldots & 0 \\
0 & 0 & 0 & \ldots & 0
\end{array}\right)=a E\left(\begin{array}{ccccc}
0 & 1 & 0 & \ldots & 0 \\
0 & 0 & 0 & \ldots & 0 \\
\ldots & \ldots & \ldots & \ldots & 0 \\
0 & 0 & 0 & \ldots & 0
\end{array}\right) \in(a E) \subset(A) \subset N\left(R_{n}\right)
$$

Therefore $B$ is strongly regular: $B=B^{2} Y$. But since $B^{2}=0$, we have $B=0$, $a=0$, and $A=0$.

When $R$ does not possess a unit element, we can obtain a ring $\hat{R}$ in the usual way by adjoining a unit element to $R$. Then $R_{n}$ is an ideal of $\hat{R}_{n}$, and $N\left(R_{n}\right)=R_{n} \cap N\left(\hat{R}_{n}\right)=0$.

The above theorem shows that there exists a ring $R$ such that $M(R) \varsubsetneqq N(R)$.

THEOREM 6. $M(R)=N(R)$ if and only if $M(R)$ has no non-zero nilpotent element.

Proof. Suppose that $M(R)$ has no non-zero nilpotent element. Then, since for every $a \in M(R)$ there is an $x$ such that $a=a x a$ whence $\left(a-a^{2} x\right)^{2}=0$, we have $a=a^{2} x, a \in N(R)$. This means $M(R)=N(R)$. The converse follows from Lemma 4.

COROLLARY. If $R$ is either commutative or has no non-zero nilpotent element, then $M(R)=N(R)$.

\section{REFERENCES}

[1] B. Brown and N. H. McCoy, The maximal regular ideal of a ring. Proc. of the Amer. Math. Soc. 1 (1950), pp. 165-171.

[2] R. F. Arens and I. Kaplansky, Topological representation of algebras. Trans. Amer. Math. Soc. 63 (1948), pp. 457-481.

Mathematical Institute, Nagoya University 